\title{
Characteristics of Flame Propagation in a Vortex Flow for Deflagration-to-Detonation Transition
}

\author{
By Katuo Asato, Takeshi MiYasaKa, Takuya SuKEgawa, \\ Kouki TANABASHI, Atsuhiro KaWAMATSU and Yasuaki KozATO \\ Department of Human and Information Systems, Gifu University, Gifu, Japan
}

(Received June 24th, 2013)

\begin{abstract}
Behaviors of flame propagation in a detonation tube were observed by the schliren system with a high-speed video camera, and the flow velocity, flame velocity and propagation velocity of the pressure wave and the maximum pressure were measured to clarify the combined effects of a vortex flow on flame propagation and the deflagration-to-detonation transition (DDT) process. The flame is accelerated and the flame velocity is rapidly increased when the Shchelkin spiral is inserted in the tube. The accelerating flame acts as a piston producing the compression wave and it proceeds to the shock, then transition to detonation is accomplished. The DDT distance in the case with the Shchelkin spiral becomes shorter due to the combined effects of a VF and Shchelkin spiral.
\end{abstract}

Key Words: DDT, Vortex Flow, Flame Observation, Flame Velocity, Pressure Wave Velocity

\section{Introduction}

Pulse detonation engines (PDEs) are expected to be a new power source for aerospace vehicles and electric generators because of the higher thermal efficiency of a PDE compared to that of a Brayton cycle gas turbine ${ }^{1-3)}$. A key problem in PDE application is to achieve reliable and repeatable detonations in the shortest distance possible to minimize the system weight. Some authors have described using a predetonator to achieve a shorter deflagration-to- detonation transition (DDT) distance ${ }^{4,5}$. However, the advantages of PDEs, such as their simplicity and low weight, are lost if devices such as a predetonator and an additional igniter are added. To control the DDT distance without using additional ignition energy and adding sophisticated, heavy structures such as a predetonator, efforts have been undertaken to apply the vortex flow (VF) injection concept to the injection part of a PDE, because the flame can propagate very quickly in a VF, depending on the rotation velocity ${ }^{6-10)}$. In these studies, the DDT distance was shortened by $15-43 \%$, compared with that

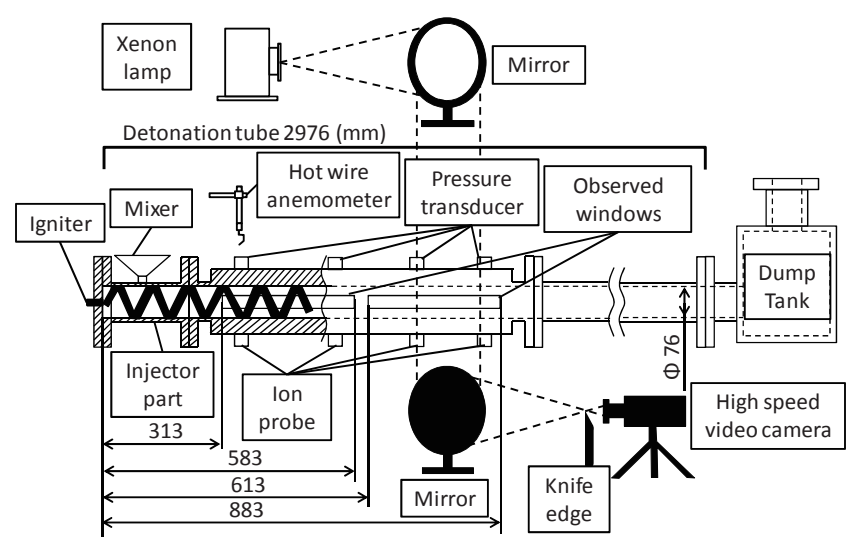

Fig. 1. Experimental apparatus. of a counterflow injector, when a VF injector was used. The shortening effect becomes remarkable as the circumferential velocity increases ${ }^{10)}$. Turbulence in a VF promotes flame acceleration and shortens the DDT distance ${ }^{11-14)}$. In this study, the behavior of flame propagation in a detonation tube was observed, and the flame velocity, flow velocity and propagation velocity of the pressure wave and the maximum pressure were measured to clarify the combined effects of a VF and Shchelkin spiral on flame propagation and the DDT process.

\section{Experimental Apparatus}

Figure 1 show a schematic of the experimental setup, including the mixture supply, ignition system, measurement system and dump tank. An observation window was installed at the downstream location between $\mathrm{x}=313 \mathrm{~mm}$ and $\mathrm{x}=883$ $\mathrm{mm}$ from the igniter. The mixture was supplied for 3.5 or 5 second into the detonation tube (diameter $(\mathrm{d})=76 \mathrm{~mm}$ ) using an electromagnetic value. Just after supplying the mixture, the mixture was ignited by the igniter installed at the closed end of the tube. The filling rate of the mixture was $90 \%$ of the detonation tube volume. The flame was propagated in the tube, and the burned gas was exhausted into the dump tank. The pressure in dump tank is atmospheric one because the upper and lower outlets of the tank are open for the atmosphere. The burned gas was swept by air supplied from the compressor after each detonation achieved propagation. The flame propagation process was observed using the Schlieren system with a high-speed video camera and ten pressure transducers and ion probes located at about 200-mm intervals. A hot-wire probe was inserted from a port in the pressure transducer, and the flow velocity in the tube was measured by a hot-wire anemometer.

Copyright $\odot 2014$ by the Japan Society for Aeronautical and Space Sciences and ISTS. All rights reserved. 


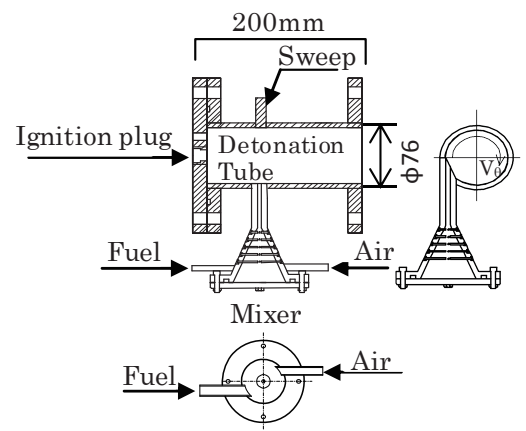

Fig. 2. Structure of vortex-flow (VF) type injector.

Figure 2 shows the schematic structure of the VF injector. There were many protuberances inside the mixer tube to promote the mixing of the fuel and oxidizer, which were supplied from the circumferential direction of the mixer tube. The mixture in the mixer tube was injected into the detonation tube in the circumferential direction of the detonation tube, establishing a VF field in the detonation tube. The Shchelkin spiral was inserted between $\mathrm{x}=0 \mathrm{~mm}$ and $\mathrm{x}=520 \mathrm{~mm}$ in this experiment. The length (Lss) and blockage ratio (BR) of the Shchelkin spiral were Lss $=520 \mathrm{~mm}$ and BR $=40.4 \%$, respectively. Experiments both with and without the Shchelkin spiral were carried out. The effects of only VF and the combined effects of a VF and the Shchelkin spiral on the DDT process were examined. Hydrogen and air (equivalence ratio $\Phi=1.0$ ) were used as the fuel and oxidizer, respectively.

\section{Experimental Results and Discussion}

\subsection{Flame propagation without the shchelkin spiral}

Figure 3 shows direct photographs, shadowgraphs and Schlieren photographs of flame propagation taken by a high-speed video camera. The front view of the white image in the direct photographs is assumed to be a flame front in this experiment. The flame front in the shadowgraphs and the Schlieren photographs can be easily described by the density variation in front of and behind the flame. The flame at the upper and lower parts of the observation windows is fast propagated compared with that at the central part of the windows. Similar flame propagation behaviors were observed in the shadowgraphs and the Schlieren photographs. The flame velocity, $\mathrm{V}_{\mathrm{fp}}$, can be obtained from variation of flame location.

Figure 4 shows variation of flame velocity without the Shchelkin spiral under the condition of the circumferential velocity at the inlet of mixture $\left(\mathrm{V}_{\theta 0}\right)=131 \mathrm{~m} / \mathrm{s}$. The circumferential velocity $V_{\theta 0}$ at the inlet of the detonation tube was obtained from the flow rate of the mixture and the cross section of the inlet tube. The abscissa, $\mathrm{x}$, shows the distance from the closed end of the tube. Four kinds of flame velocity are shown in Fig. 4. One of them is $V_{f}=\Delta l / \Delta t_{f}$, which is calculated from the distance between two neighboring ports, $\Delta \mathrm{l}$, and the differential between the arrival times of the flame at the two neighboring ports obtained from the ionization current signals, $\Delta \mathrm{t}_{\mathrm{f}}$. The others are flame velocities obtained from the direct photographs, shadowgraphs and Schlieren photographs. After ignition, the flame was propagated at about

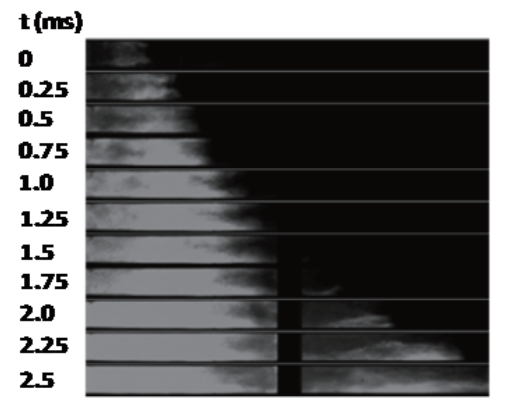

(a) Direct photographs

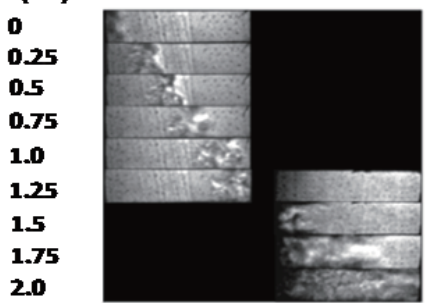

(b) Shadowgraphs

$t(\mathbf{m}$

0

0.128

0.256

0.384

0.512

0.64

0.768

0.896

1.024

1.152

1.28

1.408

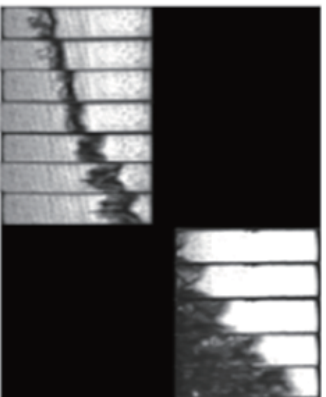

(c) Schlieren photographs

Fig. 3. Flame propagation. (VF, $\mathrm{V}_{\theta 0}=131 \mathrm{~m} / \mathrm{s}$ )

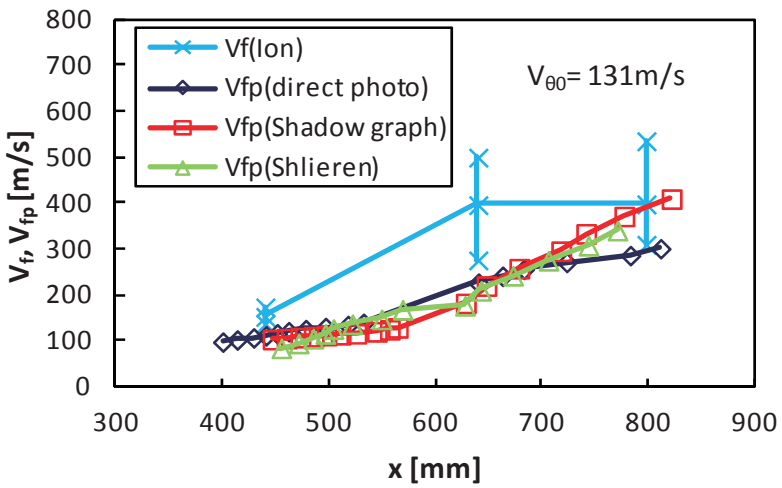

Fig. 4. Variation of flame velocity without the Shchelkin spiral.

$100 \mathrm{~m} / \mathrm{s} \sim 200 \mathrm{~m} / \mathrm{s}$ and accelerated rapidly between $\mathrm{x}=560$ $\mathrm{mm}$ and $\mathrm{x}=640 \mathrm{~mm}$ due to turbulence generated at the ramp between the upstream and downstream parts of the observation windows. The flame velocities obtained from the direct photographs are nearly the same as those obtained from the shadowgraphs and Schlieren photographs. The flame velocity calculated from the ionization current signals, $V_{f}$, was faster than the velocities obtained from the direct photographs, shadowgraphs and Schlieren photographs, $V_{\mathrm{fp}}$. This seems to indicate that the flame near the tube wall is accelerated faster than that near the central part of the tube, as shown in Fig. 3. It can also be assumed from the velocity profile in Fig. 5 that the axial velocity near the tube wall is faster than that near the central axis. 


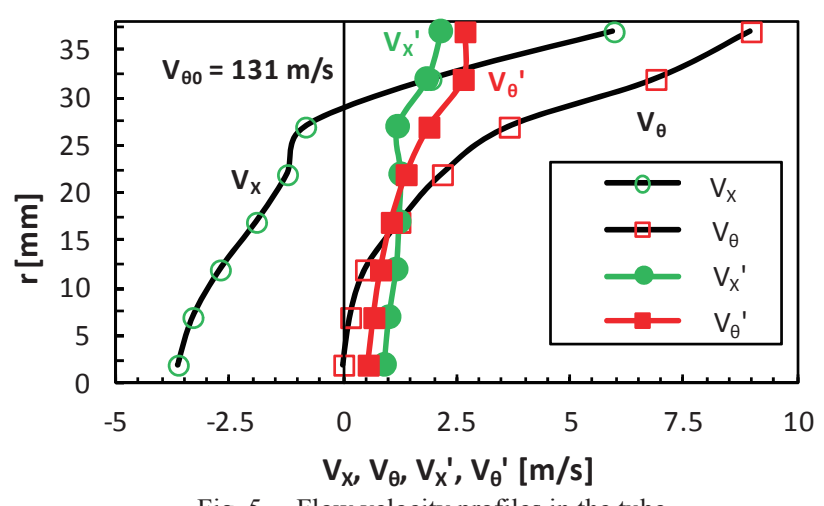

Fig. 5. Flow velocity profiles in the tube.

Figure 5 shows the flow velocity of the unburned gas at $\mathrm{x}=$ $340 \mathrm{~mm}$ in the tube, which is measured using a hot-wire anemometer. The measurement errors are $\pm 1.4 \sim 0.8 \%$ for $\mathrm{V}_{\mathrm{X}}$, $\pm 3.1 \sim 1.9 \%$ for $\mathrm{V}_{\theta}, \pm 2.6 \sim 2.8 \%$ for $\mathrm{V}_{\mathrm{X}}$ ' and $\pm 3.6 \sim 3.2 \%$ for $\mathrm{V}_{\theta}$, respectively. The vertical axis is the distance in the radial direction from the central axis of the tube. The circumferential velocity, $\mathrm{V}_{\theta}$, reaches the maximum value near the tube wall and the lowest value at the central axis of the tube. It looks like a flow field of the forced vortex. A pressure drop is generated near the central axis of the tube in such a VF, and thus a reverse flow is generated in the same place. It was confirmed using the Pitot tube and the tuft stick method. We also confirmed that the equation of continuity at a cross section of the tube was satisfied. The flow reverses in the area of $\mathrm{r}<28 \mathrm{~mm}$ and shows the positive and higher axial velocity of about $\mathrm{V}_{\mathrm{X}}=6 \mathrm{~m} / \mathrm{s}$ near the tube wall. This is why the flame near the tube wall is propagated faster than that at the central axis of the tube. On the other hand, turbulences of the axial direction $\mathrm{V}_{\mathrm{X}}$ ' and of the circumferential direction $\mathrm{V}_{\theta}$ ' are $1 \sim 2$ $\mathrm{m} / \mathrm{s}$ and $0.5 \sim 2.5 \mathrm{~m} / \mathrm{s}$, respectively. The strong turbulent flow was established in the tube. The flame propagation seems to be promoted by the turbulence.

Figure 6 shows the variation of flame velocity against the circumferential velocity, $\mathrm{V}_{\theta 0}$, at the inlet of the mixture in a $V F$. The flame velocities $V_{f}$ and $V_{f p}$ show nearly constant values even when $V_{\theta 0}$ is increased. The flame is significantly accelerated between $\mathrm{x}=440 \mathrm{~mm}$ and $\mathrm{x}=640 \mathrm{~mm}$ due to turbulence generated at the ramp between the upstream and downstream parts of the observation windows. The flame near the tube wall is propagated faster than that at the central part of the tube even when $V_{\theta 0}$ is increased.

Figure 7-(a) shows the propagation velocity, $V_{p}$, of the pressure wave. $\mathrm{V}_{\mathrm{p}}$ was calculated from the distance $\Delta \mathrm{l}$ between neighboring ports and the differential $\Delta t_{p}$ between the arrival times of the pressure wave at two neighboring ports. $\mathrm{V}_{\mathrm{p}}$ is about $400 \mathrm{~m} / \mathrm{s} \sim 900 \mathrm{~m} / \mathrm{s}$ even when $V_{\theta 0}$ is increased. The propagation velocity of the shock wave at the Chapman-Jouguet (C-J) detonation, $\mathrm{V}_{\mathrm{pC}-\mathrm{J}}$, which was calculated using the program code produced by Tanaka ${ }^{15)}$, is $1980 \mathrm{~m} / \mathrm{s}$. The measured propagation velocity is smaller than that in the C-J detonation. Figure 7-(b) shows the maximum pressure ratio, $\mathrm{P}_{\max } / \mathrm{P}_{0}$, and the differential in the arrival times of the pressure wave and the flame at each port, $\Delta \mathrm{t}_{\mathrm{N}-\mathrm{i}}$. The measured $\mathrm{P}_{\max } / \mathrm{P}_{0}$ is about $2 \sim 5$, which could not reach the Neumann spike pressure in the C-J detonation, $\mathrm{P}_{\mathrm{NC}-\mathrm{J}} / \mathrm{P}_{0}{ }^{15}$. Since $\Delta \mathrm{t}_{\mathrm{N}-\mathrm{i}}$ is about $0.5 \mathrm{~ms} \sim 1.4 \mathrm{~ms}$, the flame is also

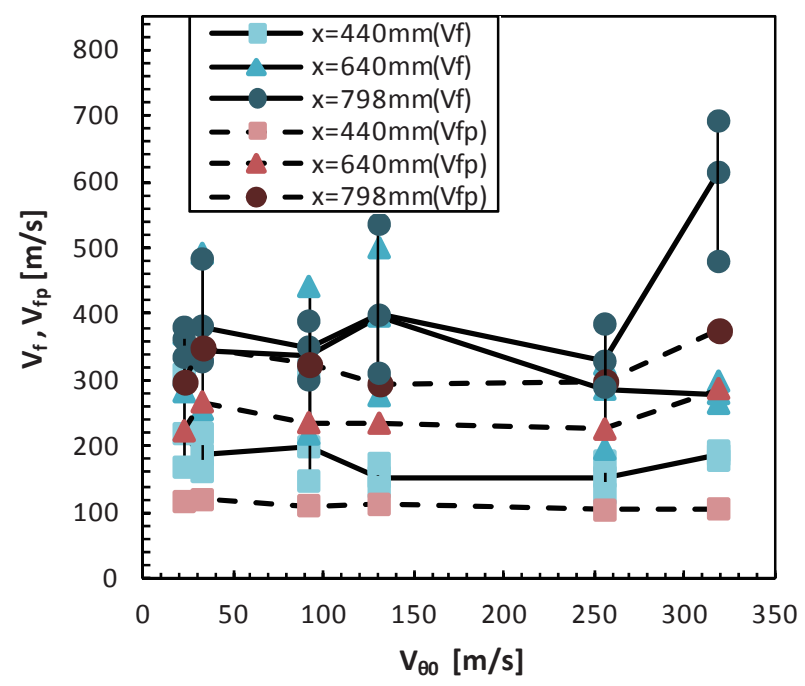

Fig. 6. Variation of flame velocity. (VF type injector)

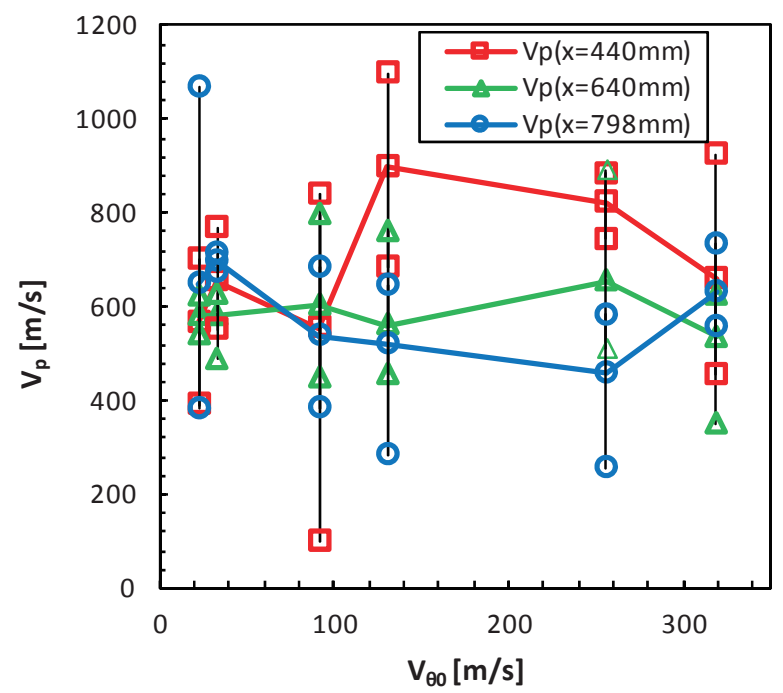

(a) Propagation velocity of pressure wave.

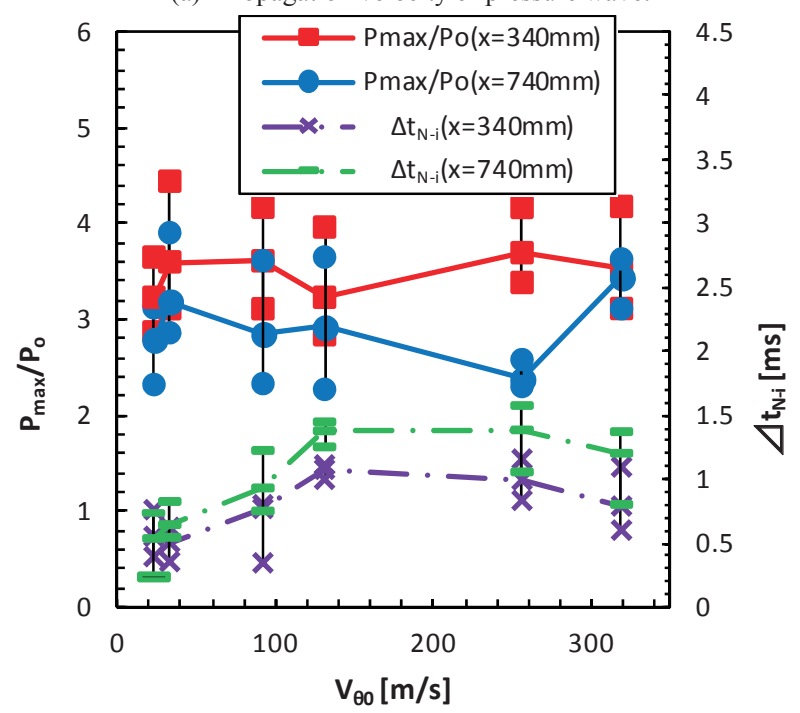

(b) Maximum pressure and the time lag between arrivals of flame and pressure wave

Fig. 7. Propagation velocity of pressure wave, maximum pressure and time lag between arrivals of flame and pressure wave.

propagated far behind the pressure wave. The results of $\mathrm{P}_{\max } /$ $\mathrm{P}_{0}, \mathrm{~V}_{\mathrm{p}}$ and $\Delta \mathrm{t}_{\mathrm{N}-\mathrm{i}}$ show that the deflagration did not change to 
the detonation when the Shchelkin spiral was not inserted in the tube. The combined effects of the VF and the Shchelkin spiral are important for DDT.

\subsection{Flame propagation with the shchelkin spiral}

Figure 8-(a) shows the Schlieren photographs of flame propagation with the Shchelkin spiral. The field of vision is between $\mathrm{x}=313 \mathrm{~mm}$ and $\mathrm{x}=583 \mathrm{~mm}$ at the upstream part of the observation windows. Some white line patterns, which are indicated by the arrows and appear to be the pressure wave, can be seen in the space of the Shchelkin spiral, as show in Figs. 8-(a)-(5) (7). The flame, which is shown as the black image, appears behind the white line patterns in Figs. 8 -(a)-(5) (7). The flame joins the white line patterns at the end of the Schelkin spiral in Fig. 8-(a)-(11), and then the flame and white line patterns propagate together, as shown in Figs. 8-(a)-(11) (14).
Figure 8-(b) shows the variation of the pressure and ionization current signals with the elapsed time at port $1(\mathrm{x}=$ $340 \mathrm{~mm})$ and port $2(\mathrm{x}=540 \mathrm{~mm})$. The numbers of yellow and black lines in Fig. 8-(b) correspond to those in Fig. 8-(a). The abscissa gives the elapsed time from when the pressure wave has reached port 1 . The pressure variation at port 2 is not observed until $\mathrm{t}=0.184 \mathrm{~ms}$. A large pressure variation is generated at $\mathrm{t}>0.184 \mathrm{~ms}$, which corresponds to Fig. 8-(a)-(11) or (12). After the flame joins the pressure wave and reaches port 2, as shown in Fig. 8-(a)-(11), a large pressure increase appears repeatedly. The ionization current signal changes suddenly at $\mathrm{t}=0.224 \mathrm{~ms}$, which corresponds to Fig. 8 -(a)-(14). When the pressure signal rises at $\mathrm{t}=0.184 \mathrm{~ms}$, the ionization current does not change yet, indicating the flame propagates far behind the pressure wave.

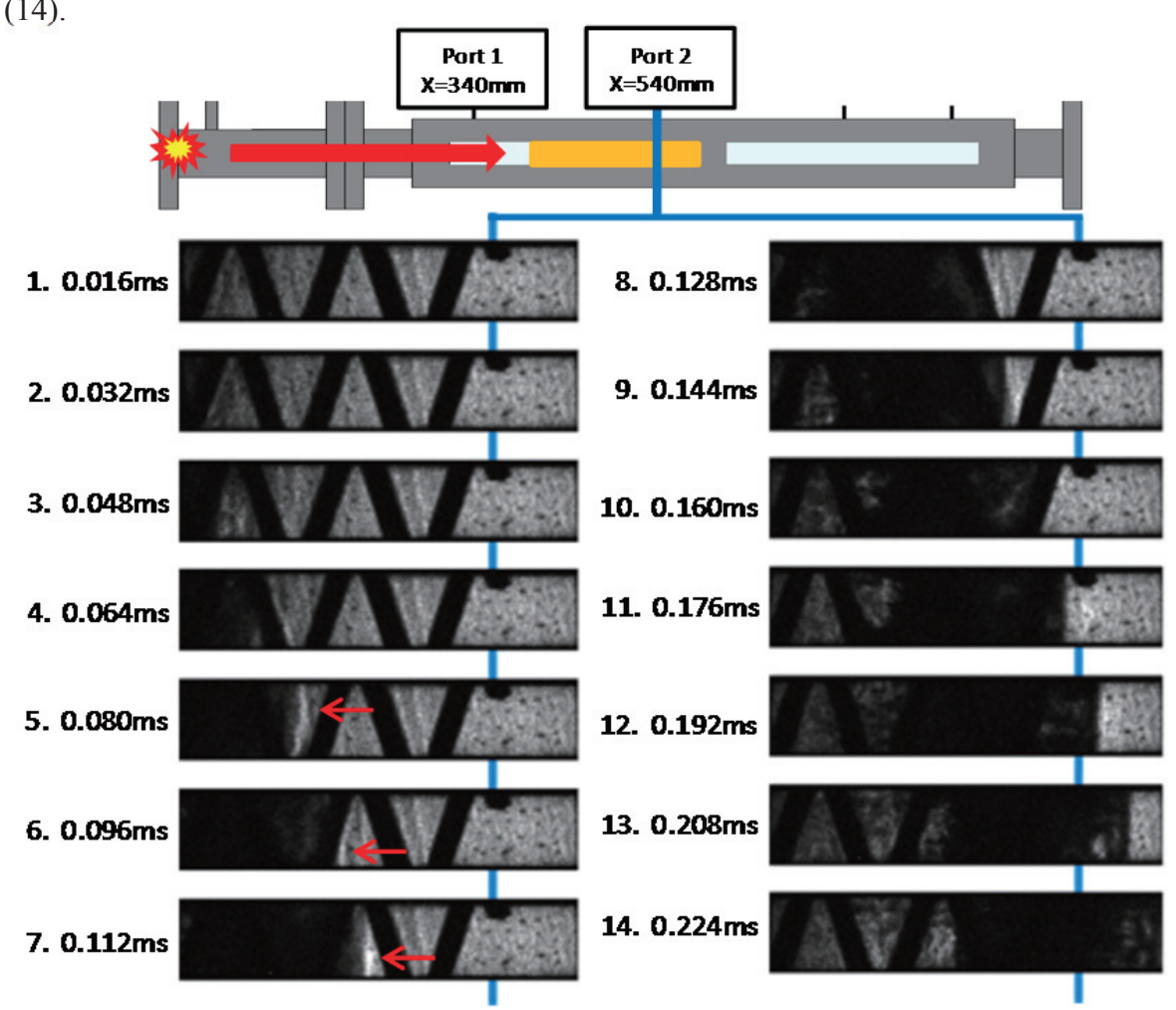

(a) Schlieren photographs between port 1 and port 2

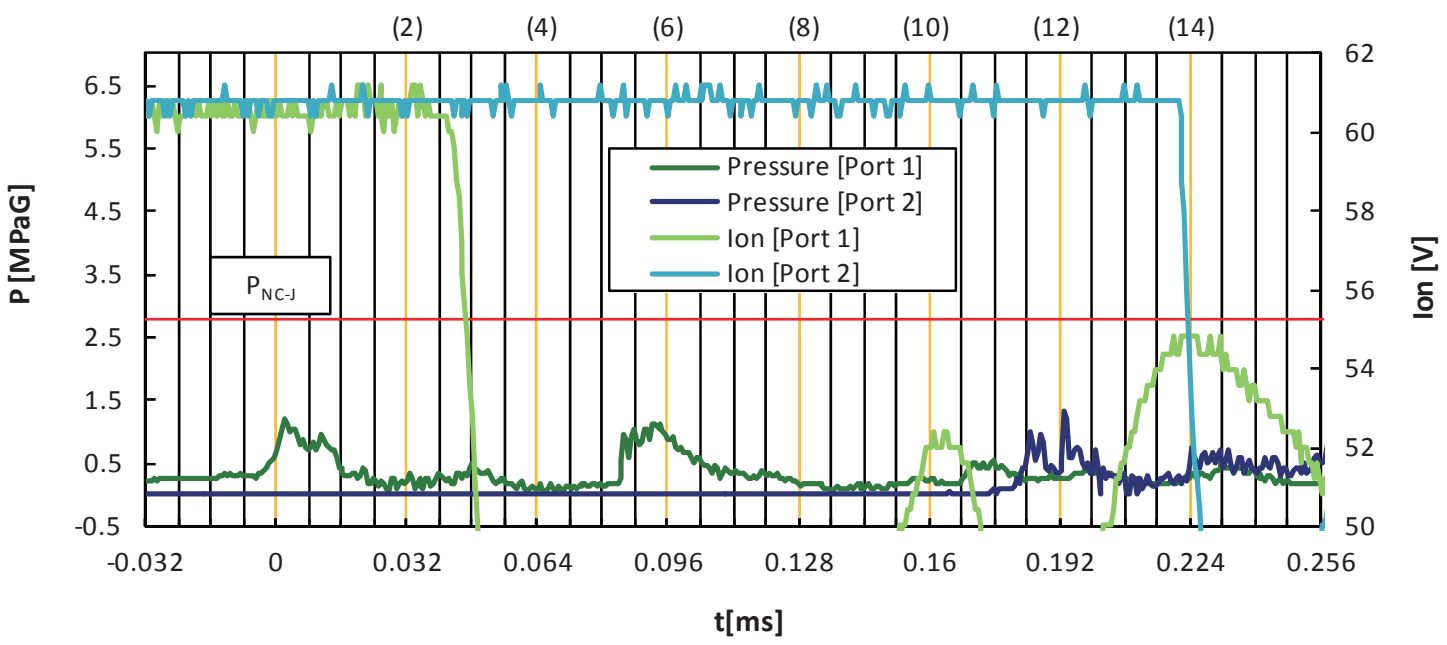

(b) Variation of pressure and ionization current signals at port 1 and port 2

Fig. 8. Flame propagation, variation of pressure and ionization current signals at port 1 and port3. 


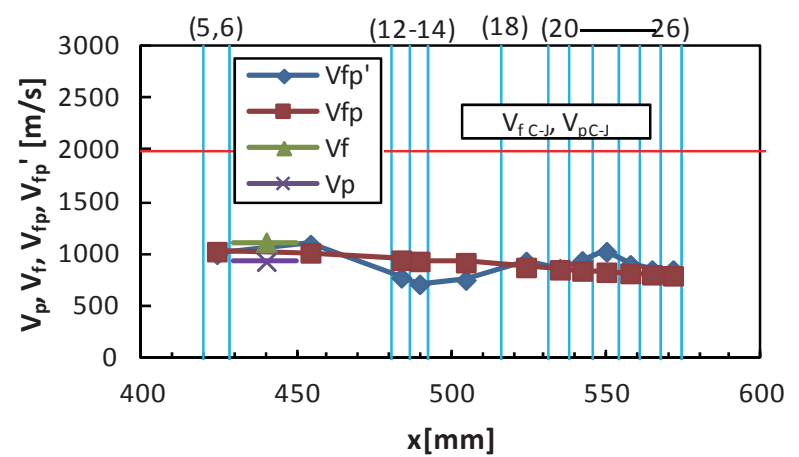

Fig. 9. Flame velocity and propagation velocity of pressure wave with the Shchelkin spiral between at port 1 and port 2.
Figure 9 shows the flame velocities $\mathrm{V}_{\mathrm{f}}, \mathrm{V}_{\mathrm{fp}}$ and $\mathrm{V}_{\mathrm{fp}}$ ' and the propagation velocity of the pressure wave, $\mathrm{V}_{\mathrm{p}}$, respectively. Here $\mathrm{V}_{\mathrm{fp}}=\mathrm{df}(\mathrm{t}) / \mathrm{dt}$ was calculated from the differential of the fitting curve $\mathrm{f}(\mathrm{t})$ of the local flame locations by the second order approximation and $\mathrm{V}_{\mathrm{fp}}{ }^{\prime}=\Delta \mathrm{l}_{\mathrm{f}} / \Delta \mathrm{t}_{\mathrm{fs}}$ was obtained using the local flame locations, $\Delta \mathrm{l}_{\mathrm{f}}$, and the time obtained from the frame rate of the high-speed-camera, $\Delta \mathrm{t}_{\mathrm{fs}} . \mathrm{V}_{\mathrm{f}}, \mathrm{V}_{\mathrm{fp}}$ and $\mathrm{V}_{\mathrm{fp}}$ ' remain nearly constant and have almost the same value as one another, but they do not reach the flame velocity of the C-J detonation ${ }^{15)}$. The propagation velocity, $\mathrm{V}_{\mathrm{p}}$, also fails to reach the value of the C-J detonation, $\mathrm{V}_{\mathrm{pC}-\mathrm{J}}{ }^{15)}$. Therefore, the flame has not changed to the detonation in this region.

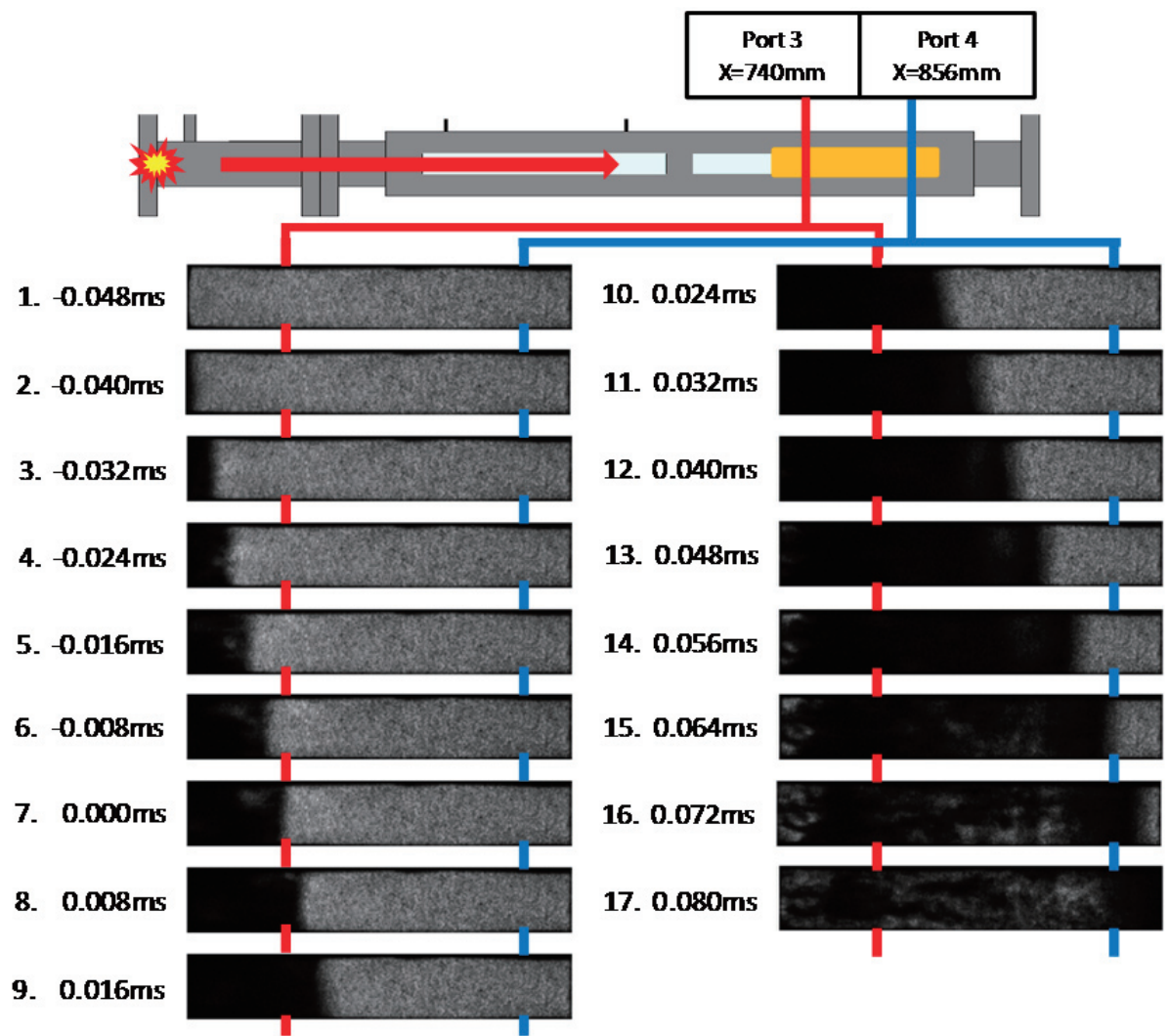

(a) Schlieren photograph between port 3 and port 4

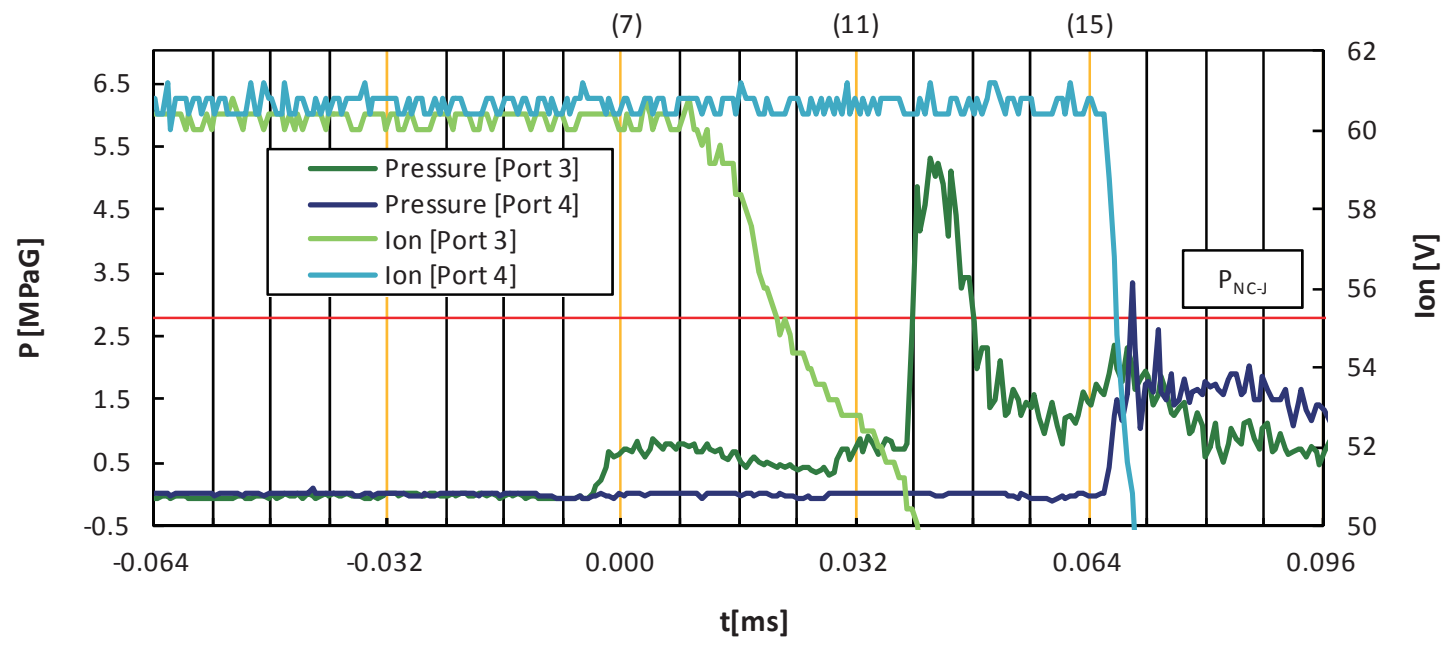

(b) Variation of pressure and ionization current signals at port 3 and port 4

Fig. 10. Flame propagation, variation of pressure and ionization current signals at port 3 and port 4 . 
Figure 10 shows the flame propagation, pressure and ionization current signal with the Shchelkin spiral. The visual field of the flame propagation is between $\mathrm{x}=613 \mathrm{~mm}$ and $\mathrm{x}=$ $883 \mathrm{~mm}$ at the downstream part of the observation windows. The pressure signal at port 3 rises at $\mathrm{t}=-0.004 \mathrm{~ms}$, which corresponds to Fig. 10-(a)-(6) or (7). The flame, however, does not reach port 3 , because the ionization current signal does not change at this time. The ionization current signal starts to change at $\mathrm{t}=0.008 \mathrm{~ms}$ which corresponds to Fig. 10-(a)-(8). The flame propagates far behind the pressure wave. The voltage of the ionization signal decreases from $60 \mathrm{~V}$ to 50 $\mathrm{V}$ at $\mathrm{t}=0.04 \mathrm{~ms}$, and then the pressure at port 3 goes above the Neumann spike pressure of the C-J detonation ${ }^{15)}$. This means that the deflagration has proceeded to the detonation near port 3 at $\mathrm{t}=0.04 \mathrm{~ms}$. The pressure and ionization current signals at port 4 suddenly change at $\mathrm{t}=0.068 \mathrm{~ms}$, which corresponds to Fig. 10-(a)-(15) or (16). The pressure at port $4(\mathrm{x}=856 \mathrm{~mm})$ has also reached the Neumann spike pressure of the C-J detonation, $\mathrm{P}_{\mathrm{NC}-\mathrm{J}}$, when the detonation has reached port 4 , as shown in Fig. 10-(a)-(15) or (16). The flame joins the pressure wave and then the flame and pressure propagate together.

Figure 11 shows $V_{f}, V_{f p}, V_{f p}$, and $V_{p}$ with the Shchelkin spiral between $\mathrm{x}=613 \mathrm{~mm}$ and $\mathrm{x}=883 \mathrm{~mm}$. $\mathrm{V}_{\mathrm{f}}$ is about 2100 $\mathrm{m} / \mathrm{s}$, and $\mathrm{V}_{\mathrm{fp}}$, calculated from flame location, is increased from about $800 \mathrm{~m} / \mathrm{s}$ to about $2250 \mathrm{~m} / \mathrm{s}$. This means that the flame has proceeded to the detonation in this region. The local flame velocity, $\mathrm{V}_{\mathrm{fp}}{ }^{\prime}=\Delta \mathrm{l}_{\mathrm{f}} / \Delta \mathrm{t}_{\mathrm{fs}}$, rapidly increases at $\mathrm{x}=736 \mathrm{~mm}$. The flame location of $\mathrm{x}=736 \mathrm{~mm}$ corresponds to Figs. 10-(a)-(7) $\sim$ (9). After that, the flame is rapidly accelerated between $\mathrm{x}=$ $740 \mathrm{~mm}$ and $\mathrm{x}=800 \mathrm{~mm}$, and then the deflagration has changed to the detonation in this region. $V_{p}$ is a little bit smaller than $\mathrm{V}_{\text {pC-J. }}$.

Figure 12 shows variation of $V_{f}, V_{f p}$, and $V_{p}$ against the distance of $\mathrm{x}$ in all areas of the observation windows. $\mathrm{V}_{\mathrm{f}}$ is about $1100 \mathrm{~m} / \mathrm{s}$ at $\mathrm{x}=440 \mathrm{~mm}$ and increases gradually until it reaches about $2100 \mathrm{~m} / \mathrm{s}$ at $\mathrm{x}=798 \mathrm{~mm}$. On the other hand, $\mathrm{V}_{\mathrm{fp}}$ is about $760 \mathrm{~m} / \mathrm{s} \sim 1000 \mathrm{~m} / \mathrm{s}$ and remains nearly constant between $\mathrm{x}=440 \mathrm{~mm}$ and $\mathrm{x}=600 \mathrm{~mm}$. It seems that since the flow in the tube reverses at the area of about $\mathrm{r}<28 \mathrm{~mm}$, as shown in Fig. 5, the flame is accelerated near the tube wall, and the flame near the central axis of the tube is not accelerated. After that, $V_{f p}$ is increased rapidly until about $2200 \mathrm{~m} / \mathrm{s}$ at $\mathrm{x}=832 \mathrm{~mm} . \mathrm{V}_{\mathrm{p}}$ increases until about $1700 \mathrm{~m} / \mathrm{s}$. $\mathrm{V}_{\mathrm{fp}}$ and $\mathrm{V}_{\mathrm{f}}$ reach the value of the C-J detonation at $\mathrm{x}=856$ $\mathrm{mm}$.

Figure 13 shows $\mathrm{P}_{\max } / \mathrm{P}_{0}$ and $\Delta \mathrm{t}_{\mathrm{N}-\mathrm{i}}$ at each port. The maximum pressure ratio is initially about $10 \sim 20$ and reaches about 30 at $\mathrm{x}=856 \mathrm{~mm}$, which is greater than the Neumann spike pressure of the C-J detonation, $\mathrm{P}_{\mathrm{NC}-\mathrm{J}} / \mathrm{P}_{0}=27^{15)}$. The differential in the arrival times, $\Delta \mathrm{t}_{\mathrm{N}-\mathrm{i}}$ is initially about $0.07 \mathrm{~ms}$. The flame propagates far behind the pressure wave between $\mathrm{x}$ $=340 \mathrm{~mm} \sim 600 \mathrm{~mm}$. After that, $\Delta \mathrm{t}_{\mathrm{N}-\mathrm{i}}$ becomes about $0.02 \mathrm{~ms}$ $\sim 0.01 \mathrm{~ms}$, and then the flame joins the pressure wave and they propagate together. The flame seems to proceed from deflagration to detonation at $\mathrm{x}=755 \mathrm{~mm} \sim 830 \mathrm{~mm}$, based on all the results of $\mathrm{P}_{\max } / \mathrm{P}_{0}, \mathrm{~V}_{\mathrm{f}}, \mathrm{V}_{\mathrm{fp}}$ and $\Delta \mathrm{t}_{\mathrm{N}-\mathrm{i}}$.

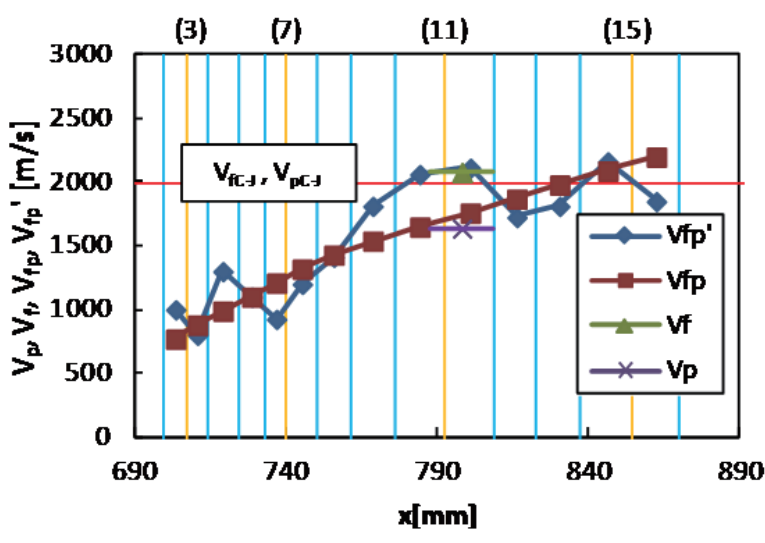

Fig. 11. Flame velocity and propagation velocity of pressure wave with the Shchelkin spiral between at port 3 and port 4 .

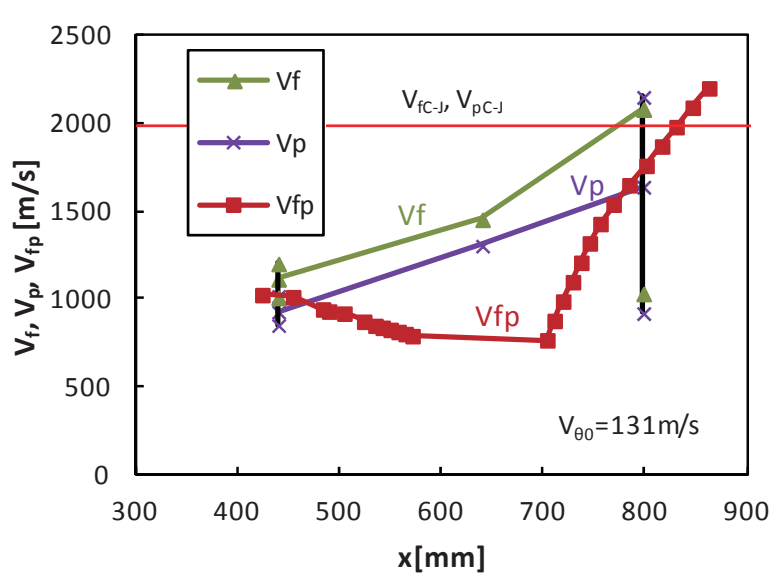

Fig. 12. Flame velocity and propagation velocity of pressure wave with the Shchelkin spiral in all areas of observation windows.

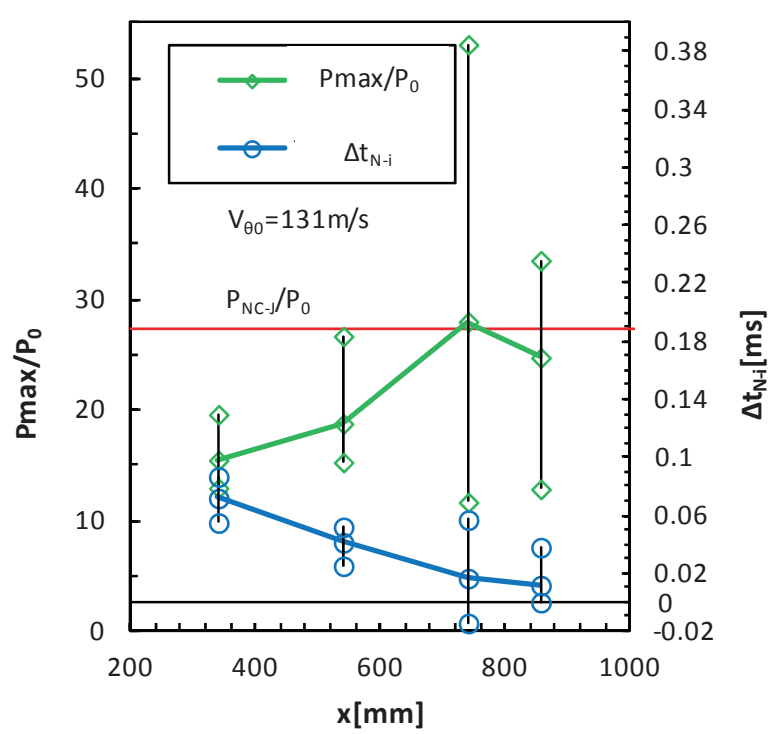

Fig. 13. Maximum pressure and time lag between arrivals of flame and pressure wave. 


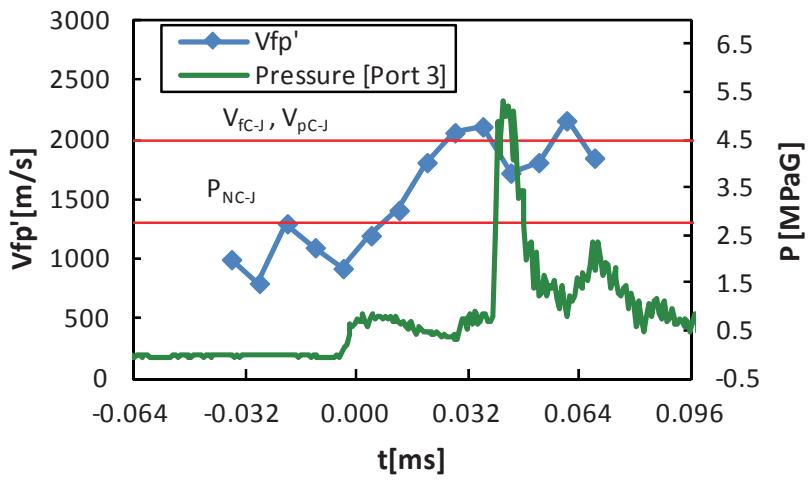

(a) With the Shchelkin spiral

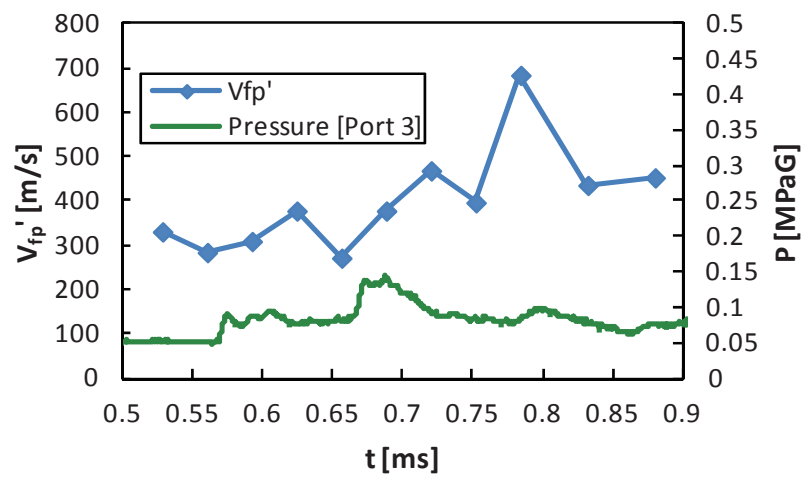

(b) Without the Shchelkin spiral

Fig. 14. Time histories of flame velocity and pressure with and without the Shchelkin spiral.

Figure 14 shows the time histories of the flame velocity $\mathrm{V}_{\mathrm{fp}}$, and the pressure variation $\mathrm{P}$ at the port 3 . The abscissa gives the elapsed time from when the pressure wave has reached the port 3. The expansion of the high temperature burned gas induces a pressure wave ahead of the flame front when the flame is initiated near the closed end of the tube. The turbulence near the tube wall is also generated when the Shchelkin spiral is inserted to the tube. The flame velocity becomes higher due to the turbulence. The higher flame velocity induces the expansion of the unburned mixture in the higher pressure. This positive feedback coupling is established between $\mathrm{t}=-0.032 \mathrm{~ms}$ and $0.032 \mathrm{~ms}$ as shown in Fig. 14-(a), then the flame velocity increases rapidly. Such feedback determines rapid flame acceleration near the port 3. The accelerating flame acts as a piston producing compression waves in the unburned mixture. The compression wave produced by the flame proceeds to the shock at about $\mathrm{t}=0.032$ $\mathrm{ms}$ and transition to detonation is accomplished. Then, the flame velocity reaches the value of the C-J detonation, as shown in Fig. 14-(a).

On the other hand, when the Shechelkin spiral is not inserted to the tube, the large turbulence in the tube is not generated and the flame acceleration due to the piston effects by the accelerating flame is smaller than that in the case with the Shchelkin spiral. The compression wave is not proceeded to the shock, then the flame velocity does not reach the value of the C-J detonation, as shown in Fig. 14-(b).

\section{Conclusions}

Behaviors of flame propagation in the detonation tube were observed, and the flow velocity, flame velocity and propagation velocity of the pressure wave and the maximum pressure were measured to clarify the combined effects of a VF and the Shchelkin spiral on flame propagation and the DDT process. The following results were obtained;

The flame is accelerated and the flame velocity is rapidly increased when the Shchelkin spiral is inserted in the tube. The accelerating flame acts as a piston producing the compression wave and it proceeds to the shock, then transition to detonation is accomplished. The DDT distance in the case with the Shchelkin spiral becomes shorter due to the combined effects of a VF and the Shchelkin spiral than that is the case without the Shchelkin spiral.

\section{References}

1) Wintenberg, E. and Shepherd, J. E.: Thermodynamic Cycle Analysis for Propagating Detonation, J. of Propulsion and Power, 22 (3) (2005), pp. 694-697.

2) Wu, Y., Ma, F. and Yang, V.: System Performance and Thermodynamic Cycle Analysis of Air Breathing Pulse Detonation Engines, J. of Propulsion and Power, 19 (2003), pp. 556-560.

3) Miyasaka, T., Fujiwara, T., Endo, T., Zhang, F.-Y. and Matsuo, T.: Estimation of Pulse Detonation Engine Performance Using a Two-Dimensional CFD Analysis Based on Detailed Oxyhydrogen Chemistry, Trans of the Japan Society for Aeronautical and Space Science, 50 (2002), pp. 10-15.

4) Frolov, S. M., Basevich, V. Y. and Aksenov, V. S.: Detonation Initiation by Controlled Triggering of Electric Discharages, J. of Propuls. Power, 19 (4) (2003), pp. 573-580.

5) Frolov, S. M., Basevich, V. Y. and Aksenov, V. S.: Combined Strategies of Detonation Initiation in a Liquid-Fueled Air Breathing PDE, Proc. of Int. Symp. on Air Breathing Engine, (2005), CD-ROM 2005-1292, pp. 1-7.

6) McCormack, P. D., Scheller, K., Mueller, G. and Tisher, R.: Flame Propagation in a Vortex Core, Combust. Flame. 19: 297 (1972), pp. 297-303.

7) Asato, K., Wada, H., Hiruma, T. and Takeuchi, Y.: Characteristics of Flame Propagation in a Vortex Core: Validity of a Model for Flame Propagation. Combust. Flame. 110 (1997), pp. 418-428.

8) Ishizuka, S., Murakami, T., Hamasaki, T., Koumura, K. and Hasegawa, R.: Flame Speed in Combustible Vortex Rings. Combust. Flame. 133 (1998), pp. 542-553.

9) Asato, K., Miyasaka, T. and Kitoh, Y.: Effect of Vortex Flow Injection on Shortening the Distance of Deflagration-to-Detonation Transition, Deflagration and Detonation Combustion, Torus press, Moscow, (2010) pp. 347-360.

10) Asato, K., Miyasaka, T., Watanabe, Y., Ishikawa, S. and Tanabashi, K.: Effects of a Vortex Flow on Characteristics of Deflagration-to-Detonation Transition, Trans. JSASS Aerospace. Tech. Japan, 10 (28) (2012), pp. 15-20.

11) Oppenheim, A. K., Manson, N. and Wagner, H. G.: Recent Progress in Detonation Research. AIIAAJ, 1 (1963), pp. 2243-2252.

12) Montgomery, C. J, Khokhlov, A. M. and Oran, E. S.: The Effect of Mixing Irregularities on Mixed-Region Critical Length for Deflagration-to-Detonation Transition, Combust. Flame. 115 (1-2) (1998), pp. 38-50.

13) Lee, S. Y., Watts, J., Saretto, S., Pal, S., Conrad, C., Woodward, R. and Santoro, R.: Deflagration to Detonation Transition Processes by Turbulence-Generating Obstacles in Pulse Detonation Engines, J. of Propuls. Power. 20 (6) (2004), pp. 1026-1036.

14) Li, J., Lai, W. H., Chung, K. and Lu, F. K.: Experimental Study on Transition of an Overdriven Detonation Wave from Propane/Oxygen to Propane/Air, Combust. Flame, 154 (3) (2008), pp. 331-345.Tanaka K.

15) http://riodb.ibase.aits.go.jp/ChemTherm/aistjan.html 